\title{
Lung Cancer: Biomarkers, Tyrosine Kinase Inhibitors and Monoclonal Antibodies
}

\author{
Made Putra Semadhi ${ }^{1,2}$, Stefanus Layli Prasojo ${ }^{1,2}$, Anandani Widarini ${ }^{1,3}$ \\ ${ }^{1}$ Faculty of Pharmacy, Padjajaran University, Bandung, Indonesia \\ ${ }^{2}$ Prodia Clinical Laboratories, Jakarta, Indonesia \\ ${ }^{3}$ Prodia Occupational Health Institute, Jakarta, Indonesia
}

Lung cancer is the most contributor of cancer cause death in the world. Lung cancer is related to cigarette consumption and genetic factor. Nicotine derived nitrosamine ketone is the most important inducer of lung cancer associated with DNA Mutations resulting in the activation of Kirsten rat sarcoma viral (KRAS) oncogenes. DNA Mutation in Lung cancer is mostly presence by epidermal growth factor receptor (EGFR) mutations. There were seven potential biomarkers to detect early lung cancer, whereas carcinoembryonic antigen (CEA), neuron specific enolase (NSE), cytokeratin-19 fragments (CYFRA 21-1), alpha-fetoprotein (AFP), cancer antigen 125 (CA-125), CA-199 and ferritin. The use of biomarkers in combination can improve the accuracy in diagnosing lung cancer. Other biomarkers include KRAS mutations, B-type Raf kinase (BRAF) mutation, mesenchymal-epithelial transition factor (MET) amplification and Excision repair cross-complementing group 1 (ERCC1) can be used to see whether there are any genetic mutations that lead to lung cancer. Treatment of lung cancer with chemotherapy can be done using tyrosine kinase inhibitors and monoclonal antibodies.

Keywords: lung cancer, DNA mutation, EGFR, KRAS, BRAF, MET, tyrosine kinase

\section{Introduction}

The signaling pathway of epidermal growth factor (EGF) receptor (EGFR), is activated in more than half of the patients with non-small cell lung cancer (NSCLC). This activation can be a result of overexpression of the protein, the increasing number of genes copies or genetic mutation. Estrogen receptor binding (ErbB) receptor family consists of four receptor tyrosine kinases: EGFR, also called ErbB1 or human epidermal receptor (HER)1; ErbB2 (HER2/neu), ErbB3 (HER3), and ErbB4 (HER4). ${ }^{1,2}$ Insufficient ErbB signaling in humans is associated with the development of neurodegenerative diseases, such as multiple sclerosis and Alzheimer's disease.

Date of submission: March 31, 2017

Accepted for publication: May 25, 2017

\section{Corresponding Author:}

Made Putra Semadhi

Faculty of Pharmacy, Padjajaran University

Jl. Eijkman No.38, Bandung, Indonesia

E-mail: putra.semadhi@gmail.com
Binding of receptor and ligand induces receptor dimerization, resulting in the phosphorylation of tyrosine residues in the kinase domain. ${ }^{3,4}$ These phosphotyrosines recruit partner proteins that trigger intracellular signaling cascades, chiefly through the mitogen-activated protein kinase (MAPK) and phosphatidylinositol 3-kinase (PI3K) pathway. ${ }^{5}$ This pathway is involved in the induction of cell proliferation, protection from apoptosis, ${ }^{6,7}$ activation of angiogenesis and development of metastasis. Erlotinib and gefitinib were developed as reversible and highly specific small-molecule tyrosine kinase inhibitors that competitively block the binding of adenosine triphosphate to its binding site in the tyrosine kinase domain of EGFR. ${ }^{8}$ Erlotinib and 
gefitinib seem to be very effective in treating tumors with gene mutations that activate EGFR. The tumor may depend on the activity of the EGFR pathway for their survival. ${ }^{9}$

EGFR is single chain transmembrane glycoproteins consisting of an extracellular ligand-binding ectodomain, a transmembrane domain, a short juxtamembrane section, a tyrosine kinase domain and a tyrosine-containing C-terminal tail. The EGFR tyrosine kinase regulates cell proliferation and survival through the automatic activation of EGFR itself or two pathways mediating inter downstream: the phosphatidylinositol-4,5-bisphosphate 3-kinase catalytic subunit alpha (PIK3CA) / Akt1 / mammalian target of rapamycin (mTOR) pathway and the Ras / Raf / MAPK pathway. The ligand-binding induced dimerization of tyrosine residues in the cytoplasmic domains. ${ }^{10}$ This activation of EGFR initiates signaling cascades which induce critical cellular responses, such as proliferation, differentiation, motility and survival.

\section{EGFR alterations in lung cancer}

A comprehensive literature review indicated that 569 mutations were found in 2,880 lung cancer patients. Mutation distribution of EGFR is as follows: $48 \%$ in exon $19 ; 43 \%$ in exon $21 ; 4 \%$ in exon 20 and $3 \%$ in exon 18 . EGFR mutations, except EGFRvIII, are rarely found in the squamous cell and large cell carcinomas. Thus tyrosine kinase inhibitor (TKI) therapy may not be a relevant therapy for patients with those tumors. In contrast, EGFR mutations were found in $10 \%$ of 375 adenocarcinomas and $26 \%$ of the 86 cases designated as bronchioloalveolar carcinomas. ${ }^{11}$

Mutations with constitutive activation of the receptor tyrosine kinase are often associated with either sensitivity or resistance to EGFR TKI. ${ }^{12}$ The response-associated mutations are linked with response rates of $47 \%$ in patients treated with either erlotinib or gefitinib. ${ }^{13}$ EGFR mutations of exons 18-24 in tumors from 10 gefitinib-responsive and 7 erlotinib-responsive demonstrated that EGFR mutations were present in 7 of $10(70 \%)$ gefitinib-responsive and in 5 of $7(71 \%)$ erlotinib-responsive tumors. ${ }^{14}$

\section{Treatment}

Guidelines for the diagnostic with known or suspected lung cancer include obtaining a thorough history, physical examination and appropriate elaboration tests to screen for metastatic disease. Type of lung cancer confirmation by cytology is important before treatment can be determined. ${ }^{15}$

\section{NSCLC}

Surgery is the treatment of choice for individuals with early-stage NSCLC. The type of surgical procedure depends on tumor location, the patient's comorbidities and the potential effects on long-term pulmonary function. Patients with positive surgical margins were evaluated for additional treatment, which typically includes a radiation/ chemotherapy. Radiation therapy is the main treatment for people who have comorbidities, such as heart disease or severe lung disease, or who are poor surgical candidates. ${ }^{16}$ Patients with complete resection and those with residual nodal disease found at surgery, may be considered for postoperative radiation therapy. Healthy IIIB patients with clinical T4N0 of satellite tumor in the same lobe or involvement carinal should be referred to a thoracic surgeon for possible resection. People with stage IV NSCLC have a poor prognosis with a median survival of 8-10 months and 1-year survival rate of $30-35 \%$. $^{15,16}$

\section{Small cell lung cancer (SCLC)}

SCLC usually is more aggressive than NSCLC. Distant metastasis is common at initial presentation. Primary treatment usually consists of combination chemotherapy, which may be combined with radiation therapy. Limitedstage disease can potentially be cured with combination chemotherapy and radiation therapy. Surgical resection may be considered in rare situations when a patient has limited SCLC. Around $50-60 \%$ of the patients who achieved a complete response after chemotherapy induction will develop brain metastases within 2 years. The brain is the only site of metastasis in $20-30 \%$ of these patients. Consequently, people who achieve complete remission are offered prophylactic cranial irradiation to decrease the risk of central nervous system involvement. If patients relapse after an initial response to treatment or if they do not respond to initial treatment, further chemotherapy is usually offered, depending on the patient's previous response to first-line therapy. In patients with extensive disease, radiation therapy may be used for palliation. ${ }^{15,16}$

\section{Chemotherapy}

NSCLC management diagnosed until the disease has spread beyond the primary site; approximately $55 \%$ of patients have the metastatic (stage IV) disease at diagnosis. ${ }^{17}$ For these patients, chemotherapy is the foundation of their treatment and critical in determining their survival and quality of life. Platinum-based therapy is the mainstay of chemotherapy for NSCLC and is usually given in combination with a tubulin 
binding agent (TBA); including the taxanes (paclitaxel, docetaxel), vinca alkaloids (vinorelbine, vincristine), a camptothecin analog (irinotecan, topotecan), gemcitabine, or pemetrexed. Despite an expanding panel of chemotherapy agents and emerging data regarding the most effective ways to deploy such agents, tumor cell resistance to chemotherapy agents continues to pose a significant challenge in the management of human neoplasms.

Chemoresistance may be innate or acquired and may apply to a single agent or a class of agents with the same/similar antineoplastic mechanisms of action. Chemoresistance is a multifaceted problem with diverse clinical manifestations that requires an understanding of both basic mechanisms and evolution of such resistance as cancer progress. Active efflux of chemotherapeutic agents is achieved via ATP-binding cassette (ABC) transporters (including P-glycoprotein [P-gp] and multi-drug resistance proteins [MDRs]). This mechanism contributes to resistance to anthracyclines, taxanes, platinum agents, vinca alkaloids and topoisomerase inhibitors. Modification of the drug target, including mutation of the binding site or in the case of TBAs, has a change in the relative proportion of the various tubulin isoforms, mediate resistance to taxanes, antifolates and topoisomerase inhibitors. Changes or mutations in the mitotic checkpoint signaling, allowing tumor cells to bypass or overcome that exert antimitotic agent/proapoptotic effects, mediating resistance to platinum agents, taxanes and anthracyclines.

Chemoresistance is common in NSCLC. In a study, extreme or intermediate resistance to carboplatin was documented in $68 \%(1056 / 1565)$ of samples and cisplatin resistance was in $63 \%(1409 / 2227)$ of samples. ${ }^{18}$ Resistance to doxorubicin, etoposide, gemcitabine, vinorelbine, paclitaxel, docetaxel and topotecan was reported in 75 , $63,72,42,40,52$ and $31 \%$ of samples, respectively. ${ }^{18}$ All NSCLC patients will eventually develop resistance to the chemotherapeutic agents to which they are exposed, even with a good initial response, and most will receive two or three lines of therapy. The challenge in managing patients with NSCLC is the need to establish a long-term view, taking into account the likelihood of developing chemoresistance and the effect of using an agent at each line of therapy. Currently, there is no reliable strategy available to guide treatment selection from first-line through all subsequent lines of therapy to avoid the development of chemoresistance, although active clinical investigations are ongoing. ${ }^{18}$
Some platinum analogs are in early clinical development including picoplatin (cisplatin analog). This agent has demonstrated clinical activity among patients with platinum-refractory SCLC in Phase II and III trials as a second-line treatment following platinum therapy. ${ }^{19}$

ABT-751 (a sulfonamide) is a novel investigational TBA that binds to the colchicines site of tubulin and inhibits the polymerization of microtubules. Early clinical data for this agent is encouraging in heavily pretreated patients, including those previously treated with platinumbased regimens. ${ }^{20}$ The epothilones, including ixabepilone, sagopilone and patupilone, are a novel class of TBAs. The epothilones bind tubulin in the same location as the taxanes. However, the molecular nature of epothilone binding is fundamentally different, involving a group of alternative amino acids. ${ }^{21}$ Importantly, the epothilones retain activity in cell lines resistant to taxanes. The epothilones appear to decrease III-tubulin expression and restore sensitivity of tumor cells to other chemotherapeutic agents including cisplatin and taxanes. In breast cancer cells, ixabepilone appears to be less sensitive to mutational changes within the III-tubulin gene that impair taxane binding, ${ }^{22}$ and better at suppressing the overall dynamicity of III-tubulin compared with paclitaxel non-taxane resistant breast cancer cells. Also, in vitro data suggests that ixabepilone may overcome taxane resistance in breast cancer cell lines through preferential binding to the III-tubulin isoform. ${ }^{23} \mathrm{~A}$ Phase II study with patupilone resulted in a response rate of $11 \%$ among 47 patients with NSCLC. The epothilones can offer an alternative for patients with congenital taxane resistance include those with pre-treatment of high-level III-tubulin.

\section{Erlotinib and gefitinib}

EGFR alterations have prompted the development of two classes of anti-EGFR agents: monoclonal anti-EGFR antibodies (such as cetuximab, panitumumab, etc.) and small molecule TKIs directed against EGFR tyrosine kinase (such as gefitinib, erlotinib, etc.). The results of the clinical trials indicated that many of the tumors harboring mutant EGFR are highly sensitive to EGFR TKIs, with up to $70 \%$ demonstrating a significant clinical response. ${ }^{24}$ Recent studies have provided more compelling evidence of the clinical benefits of anti-EGFR treatment in the appropriate setting. ${ }^{25}$ Evidence from the large phase III randomized Iressa Pan-Asia Study trial has prompted the American Society of Clinical Oncology to issue a provisional clinical opinion, recommending the testing of EGFR mutational 
status in patients being considered for first-line EGFR TKI therapy owing to their demonstrated benefit on progressionfree survival. ${ }^{26}$

Erlotinib and gefitinib have been studied in a variety of settings in clinical trials. In two parallel phase III studies, erlotinib and gefitinib were compared with placebo in patients with advanced NSCLC in whom standard chemotherapy had failed. In a National Cancer Institute of Canada Clinical Trials Group (NCIC CTG) BR.21 trial (NCT00036647), 731 patients with stage IIIB or IV NSCLC were randomly assigned to receive either erlotinib (at a daily dose of $150 \mathrm{mg}$ ) or placebo. Erlotinib was superior compared to placebo in the analysis of overall survival (6.7 vs. 4.7 months, $p=0.001)$ and progression-free survival (2.2 vs. 1.8 months, $p<0.001) .{ }^{27}$ Meanwhile, in Iressa Survival Evaluation in Lung Cancer (ISEL) trial (NCT00242801), the effects of gefitinib ( $250 \mathrm{mg}$ daily) were compared with placebo. Gefitinib was superior to placebo in terms of time to treatment failure (3.0 vs. 2.6 months, $p<0.001)$. However, no significant differences were observed in overall survival. ${ }^{27}$

Erlotinib (similar to that of gefitinib) intracellularly binding to the ATP-binding site of the tyrosine kinase domain of EGFR, which blocks the catalytic activity of the kinase, thereby inhibiting downstream signaling of the pathways responsible for cellular proliferation. The T790M mutation, one mechanism of resistance to EGFR tyrosine kinase inhibition, is detected in approximately $50 \%$ of patients who have a relapse while receiving an EGFR tyrosine kinase inhibitor. T790M causes steric hindrance of the binding of the tyrosine kinase inhibitor and promotes constitutive activation of the EGFR mutated kinase. Thus, downstream signaling occurs despite the use of a tyrosine kinase inhibitor. ${ }^{27}$

\section{Monoclonal antibody therapy}

Another target for lung cancer is the programmed death 1 (PD-1). PD-1 receptor, which is expressed on activated T cells, is engaged by PD-ligand 1 (PD-L1) and PD-L2, which is expressed by tumor cells and infiltrating immune cells. Tumor PD-L1 expression is prevalent in NSCLC, and the interaction of PD-L1/-L2 and PD inhibit activation of T-cells and promotes tumor immune escape (i.e., the mechanism by which tumor cells escape recognition and elimination by the immune system). ${ }^{28}$

Monoclonal antibodies, such as cetuximab and panitumumab, are either chimeric mouse-human fully humanized antibodies targeting the EGFR extracellular domain, leading to blockade of ligand-activated signal transduction and receptor dimerization. Panitumumab has a high affinity for EGFR. ${ }^{29}$ The binding of the antibody starts EGFR internalization and degradation, in which leads to signal termination. The treatment has shown benefit to the clinical outcome when added to chemotherapy. However, this class of treatment only inhibits ligand-dependent activation of EGFR and not auto-phosphorylation of the tyrosine kinase domain via constitutive activation. These mutations may still activate the downstream pathways, and up-regulate cell cycle progression, cell growth and angiogenesis. $^{28}$

\section{Nivolumab}

Nivolumab is a human Immunoglobulin G4 (IgG4) PD-1 immune-checkpoint inhibitor, an antibody that disrupts PD-1-mediated signaling and restores antitumor immunity. Nivolumab is PD-1 checkpoint inhibitor that demonstrated a survival benefit with improved safety profiles. Benefits were observed, regardless of the level of expression of PD-L1. Further research is needed to identify relevant biomarkers that have sufficient sensitivity and specificity to predict which patients are most likely to benefit. ${ }^{29}$

\section{Pembrolizumab}

Pembrolizumab is a human monoclonal antibody against the PD-1, which has antitumor activity in advanced NSCLC with increased activity in tumors that express PD-L1. Pembrolizumab was associated with significantly longer overall survival than chemotherapy, despite the low number of deaths observed and the potentially confounding effect of crossover from the chemotherapy group to the pembrolizumab group. ${ }^{30}$ By data from the second interim analysis, the data and safety monitoring committee recommended to stop the trial and offer the remaining patients in the chemotherapy group to take pembrolizumab. At the time of data cutoff, $35.4 \%$ of the enrolled patients had died and $43.7 \%$ of the patients in the chemotherapy group had crossed over to receive pembrolizumab. These data underscore the substantial benefit of pembrolizumab as initial therapy for advanced NSCLC with PD-L1 expression on at least $50 \%$ of tumor cells. The safety profile of pembrolizumab observed in this trial was consistent with that seen previously with pembrolizumab for the treatment of advanced NSCLC ${ }^{31,32}$ and other tumor types.

Immune-mediated adverse events (including pneumonitis) occurred more frequently in the pembrolizumab 
group than in the chemotherapy group, whereas cytopenia is more frequently in the chemotherapy group than in the pembrolizumab group; these results are consistent with the mechanism of action for each therapy. Most immunemediated events were grade 1 or 2 severity, and none led to death. However, the overall safety profile appeared to be better with pembrolizumab than with chemotherapy. ${ }^{33}$

\section{New biomarker for lung cancer diagnosis}

The tendency for tumor biomarkers to be detected in the serum in the early stages of the disease has become an area of interest for clinicians. This study aimed to evaluate the efficiency of 7 tumor biomarkers, i.e., carcinogens embryonic antigen (CEA), neuron-specific enolase (NSE), cancer antigen-125 (CA-125), cytokeratin-19 fragments (CYFRA-21-1), CA-19.9, ferritin and alpha-fetoprotein (AFP), either independently or in combination for the diagnosis of lung cancer. The observed level of CEA, NSE, CA-125, CYFRA-21-1 and CA-19.9 in patients with pathologically-confirmed lung cancer was significantly higher than in patients with benign lung diseases or control subjects. $\mathrm{CEA}+\mathrm{NSE}+\mathrm{CA}-125$ showed the highest sensitivity for small-cell carcinoma at $83.33 \%$, while the CEA+NSE+CA-125+CYFRA-21-1 showed 94.11\% sensitivity for squamous cell carcinoma. The combination of six biomarkers i.e., CEA+NSE+CA-125+CYFRA-21$1+$ CA-19.9+ferritin, indicate the sensitivity of $80.49 \%$ for adenocarcinoma. Combining biomarkers significantly help in the diagnosis of lung cancer. However, this increased sensitivity to the combination was accompanied by decreased specificity for lung cancer subtypes. Combining the right biomarker improves sensitivity and help with the diagnosis of lung cancer. ${ }^{34}$

Cumulatively, these findings suggest that a single biomarker may not provide sufficient clues for the detection of lung cancer. As cancer are heterogeneous and have distinctive genetic and epigenetic profiles, a single biomarker also cannot provide sufficient information for predicting treatment response and patient outcome. ${ }^{35}$ However, considering the complexity of tumor origin and the heterogeneity of tumor antigen expression, a combination of biomarkers represents a useful means of assessing the histologic subtypes of lung cancer. The optimal specificity for each lung cancer subtype may be achieved by the appropriate combination of biomarkers. ${ }^{34}$

Reported clinical trials have produced favorable responses using PD-1/PD-L1 blockade as monotherapy and combined with the CTLA-4 blockade. ${ }^{36}$ Identifying ideal biomarker is needed in optimizing and personalizing immunotherapy because the clinical benefit is not observed in all patients, since every patients has their own unique variations of the human genome, due to the combination of genetic variations and environment influence. ${ }^{37}$ The utility of PD-L1 expression as a biomarker has varied based on different clinical trials and immunohistochemistry (IHC) assays. Also, response to immune checkpoint inhibition is complicated by PD-L1 expression as a marker influenced by a dynamic tumor microenvironment. Currently, there is no consensus on whether the PD-L1 expression is an ideal marker for patient selection. Further studies are needed to assess the value of predictive biomarkers for patient selection and treatment response which has a promise for alternative markers, including T-cell IHC, other immunologic markers, T-cell receptor clonality and somatic mutational burden.

\section{Wild-type EGFR}

Wild-type EGFR seems to be an important marker for EGFR TKI resistance primer. Clinical trials of Iressa PanAsia Studies show that most tumors without EGFR tyrosine kinase domain mutations detected are not sensitive to gefitinib. ${ }^{38}$ Tumors with wild-type EGFR frequently harbor somatic mutations in other genes that affect key pathways in lung adenocarcinoma. Thus, the primary drug insensitivity may be linked to the absence of drug-sensitizing mutations in EGFR and are more likely to be the result of a mutation in another gene. ${ }^{39}$

\section{Kirsten rat sarcoma viral (KRAS) mutations}

KRAS has a role in the process of EGFR signaling. Activating mutations of KRAS is present in $25-35 \%$ of TKI non-responsive cases. ${ }^{40}$ EGFR and KRAS mutations are rarely detected in the same tumor, suggesting that they may perform similar functional roles in lung tumorigenesis. ${ }^{41}$ KRAS mutation is a negative predictor of response to monoclonal antibodies anti-EGFR and is also an important mechanism of resistance to TKI in lung adenocarcinoma. A meta-analysis provides empirical evidence that somatic mutations of the KRAS oncogene are a negative predictor of a very specific response to single agent EGFR TKIs in advanced lung cancer, mostly adenocarcinomas. ${ }^{42}$ In 1470 of lung cancer of 22 publications, of which 231 cases have KRAS mutations (16\%). ${ }^{43}$ The mutations were more common in adenocarcinomas compared than other histological types of lung cancer (26vs. 16\%). The objective response rate of patients with KRAS mutant is $3 \%(6 / 210)$, whereas the 
objective response rate of patients with wild-type KRAS is $26 \%$. Like most KRAS mutations were detected in codons 12 and 13 of exon 2, an alternative algorithm-screening is performed for KRAS mutation analysis, followed by EGFR mutation assay. Mutations in KRAS are primary resistance to gefitinib and erlotinib. ${ }^{40}$ KRAS mutations are almost exclusively detected in codons 12 and 13 of exon 2, resulting in EGFR independent intracellular signal transduction activation. ${ }^{44}$

\section{B-type Raf kinase (BRAF) mutations}

The $B$-Raf gene encodes a protein that has a key role downstream of KRAS in the cell signaling pathway activating important cell functions, including cell proliferation and survival. ${ }^{45}$ Both $K$-Ras and B-Raf genes are part of the signaling cascade for the EGFR family proteins. The BRAF protein is a serine/threonine protein kinase that is activated by KRAS in a GTP-dependent manner. ${ }^{46}$ Proteins of BRAF mutant increased kinase activity and can transform NIH3T3 cells. KRAS function is not required for the growth of cancer cells with the BRAF mutation. ${ }^{47}$ Among the 697 patients with lung adenocarcinoma, BRAF mutation found in 18 patients $(3 \%)$. The frequency of mutations in BRAF V600E (>50\%), G469A (39\%) and D594G (11\%) found in exon 15, 11 and 15, respectively. All patients with BRAF mutations are smokers or former smokers. BRAF main function is believed to be mediated by phosphorylation and thus activate MAPK1, MAP2K1 and MAP2K2 path. ${ }^{46}$ Mutations in BRAF have been shown to interfere the response to panitumumab or cetuximab in patients with colorectal carcinoma. BRAF mutations are found in 1-3\% of lung cancer, mostly adenocarcinomas. ${ }^{47}$ BRAF exon 15 mutations were tested on 96 paired samples of primary lung adenocarcinomas and corresponding locoregional lymph node metastases. ${ }^{48}$ BRAF mutations were observed in two patients with KRAS mutations, demonstrating the possibility of both mutations in BRAF and KRAS occurring in the same tumor.

The initial retrospective work on mutant BRAF's effect on EGFR-targeted therapy was performed on a cohort of 132 metastatic colorectal cancer patients. The results showed that none of the patients who experienced a response displayed BRAF mutations, whereas 11 of 79 (14\%) non-responders carried a BRAF V600E allele. As BRAF mutations are mutually exclusive to EGFR and KRAS mutations, it is likely to be associated with lack of response to EGFR TKIs. ${ }^{49}$

\section{Mesenchymal-epithelial transition factor (MET) amplification}

MET is a single pass tyrosine kinase receptor essential for embryonic development, organogenesis and wound healing. MET also contributes to primary and acquired resistance to EGFR TKI. MET is located on chromosome 7q21, which encodes a tyrosine kinase and hepatocyte growth factor receptor (HGFR). ${ }^{50}$ MET amplification is associated with acquired resistance to EGFR TKI through a mechanism called the kinase switch. ${ }^{51}$ Overall, MET amplification has been reported in approximately $20 \%$ of tumors from patients with resistance which is obtained. MET amplification occurs in both squamous cell carcinoma and adenocarcinoma. ${ }^{52}$ In an in vitro study, MET amplification was associated with an increased concentration of phosphorylated HGFR. MET amplification correlates with poor prognosis in lung cancer study of surgical resection, including 241 adenocarcinomas, 139 squamous cell carcinoma and 67 other types of tumors. Aberrant MET signaling may have a key role in the development of acquired resistance to treatment with EGFR TKI. ${ }^{28}$ Clinical relevance of MET amplification has been investigated by examining tumor biopsies from patients who developed acquired resistance to gefitinib or erlotinib. MET status was assessed in a sample copy of re-biopsy from 18 patients with lung cancer during the development of secondary resistance following an initial partial response. ${ }^{53}$ In another study, MET amplification was identified in 9 of $43(21 \%)$ patients who have developed resistance secondary to EGFR TKIs in contrast to 2 of $62(3 \%)$ patients with EGFR mutation known sensitivities also have amplification of MET. Identification MET amplification has led to the development of HGFR-targeted TKI. ${ }^{54}$

\section{Excision repair cross-complementing group 1 (ERCC1) expression}

The significant prognostic of ERCC1 was accessed with real-time quantitative-PCR in surgical specimens from chemotherapy-naive patients. Investigational results of 1,207 lung cancer patients with the relationship between EGFR mutation status and ERCC1 gene expression indicated that EGFR mutated cancers are more likely to be categorized as ERCC1 low and, therefore, platinum sensitive. ${ }^{55}$ IHC evaluation of the ERCC1 expression in tumors from 130 patients revealed that ERCC1 was expressed in $10 \%$ of EGFR-mutated tumors and in $70 \%$ of EGFR wildtype tumors. Patients with low ERCC1 expression had a longer overall survival compared with the patients with 
high ERCC1 expression. Although most studies indicate a consistent association between ERCC1 expression level and responsiveness to cisplatin-based therapy, another study reported discordance of ERCC1 expression between primary and metastatic tumors. ${ }^{56}$

Approximately $25 \%$ of lung adenocarcinomas harbor KRAS mutations, which predict non-response to EGFR TKI therapy. Of the remaining KRAS-negative lung adenocarcinomas, B20\% harbor EGFR mutations, which are associated with responsiveness to EGFR TKI therapy. EGFR mutation negative cases may benefit from additional testing for the EML4-ALK rearrangement, which will be helpful in selecting patients potentially eligible for ALKtargeted therapy. ${ }^{57}$

\section{Conclusion}

Biomarkers that can be used to diagnose and monitor therapy include CEA, NSE, CYFRA 21-1, AFP, CA 125, CA-19.9, and Ferritin. The use of biomarkers in combination can improve the accuracy in diagnosing lung cancer. Other biomarkers include KRAS mutations, BRAF mutation, MET amplification and ERCC1 can be used to see whether there are any genetic mutations that lead to lung cancer.

\section{References}

1. Todaro GJ, DeLarco JE, Cohen S. Transformation by Murine and Feline Sarcoma Viruses Specifically Blocks Binding of Epidermal Growth Factor to Cells. Nature. 1976; 264: 26-31.

2. Herbst RS, Heymach JV, Lippman SM. Lung Cancer. N Engl J Med. 2008; 359: 1367-80.

3. Yarden Y, Sliwkowski MX. Untangling The ErbB Signaling Network. Nat Rev Mol Cell Biol. 2001; 2(2): 127-37.

4. Schlessinger J. Cell Signaling by Receptor Tyrosine Kinases. Cell. 2000; 103(2): 211-25.

5. Hendarmin L, Sandra F, Nakao Y, Ohishi M, Nakamura N. TNFalpha played a role in induction of Akt and MAPK signals in ameloblastoma. Oral Oncol. 2005; 41(4): 375-82.

6. Sandra F, Hendarmin L, Nakao Y, Nakamura N, Nakamura S. Inhibition of Akt and MAPK pathways elevated potential of TNFalpha in inducing apoptosis in ameloblastoma. Oral Oncol. 2006; 42(1): 39-45.

7. Sandra F, Matsuki NA, Takeuchi H, Ikebe T, Kanematsu T, Ohishi M, Hirata M. TNF inhibited the apoptosis by activation of Akt serine/threonine kinase in the human head and neck squamous cell carcinoma. Cell Signal. 2002; 14(9): 771-8.

8. Hanahan D, Weinberg RA. The Hallmarks of Cancer. Cell. 2000; 100(1): 57-70.

9. Zhang X, Gureasko J, Shen K, Cole PA, Kuriyan J. An Allosteric Mechanism for Activation of the Kinase Domain of Epidermal Growth Factor Receptor. Cell. 2006; 125(6): 1137-49.
10. Okabe T, Okamoto I, Tamura K, Terashima M, Yoshida T, Satoh T, et al. Differential Constitutive Activation of The Epidermal Growth Factor Receptor in Non-Small Cell Lung Cancer Cells Bearing EGFR Gene Mutation and Amplification. Cancer Res. 2007; 67(5): 2046-53.

11. Marchetti A, Martella C, Felicioni L, Barassi F, Salvatore S, Chella A, et al. EGFR Mutations in Non-Small-Cell Lung Cancer: Analysis of A Large Series of Cases and Development of A Rapid and Sensitive Method for Diagnostic Screening with Potential Implications on Pharmacologic Treatment. J Clin Oncol. 2005; 23(4): 857-65.

12. Hammerman PS, Janne PA, Johnson BE. Resistance to Epidermal Growth Factor Receptor Tyrosine Kinase Inhibitors in Non-Small Cell Lung Cancer. Clin Cancer Res. 2009; 15(24): 7502-9.

13. West H, Lilenbaum R, Harpole D, Wozniak A, Sequist L. Molecular Analysis-Based Treatment Strategies for The Management of NonSmall Cell Lung Cancer. J Thorac Oncol. 2009; 4 (9 Suppl 2): S1029-39; quiz S1041-2. doi: 10.1097/JTO.0b013e3181b27170.

14. Pao W, Miller V, Zakowski M, Doherty J, Politi K, Sarkaria I, et al. EGF Receptor Gene Mutations are Common in Lung Cancers from 'Never Smokers' and are Associated with Sensitivity of Tumors to Gefitinib and Erlotinib. Proc Natl Acad Sci USA. 2004; 101(36): 13306-11.

15. Knop CS. Lung cancer. In: Yarbro CH, Frogge MH, Goodman M, editors. Cancer Nursing: Principles and Practice. 6th ed. Boston: Jones \& Bartlett; 2005. p.1379-1413.

16. Van Cleave JH, Cooley ME. Lung cancer. In: Varricchio CG, editor. A Cancer Sourcebook for Nurses. 8th ed. Sudbury: Jones \& Bartlett; 2004. p.215-29.

17. Chen YB, Mu CY, Huang JA. Clinical Significance of Programmed Death-1 Ligand-1 Expression in Patients with Nonsmall Cell Lung Cancer: A 5-Year-Follow-Up Study. Tumori. 2012; 98(6): 751-5.

18. D'Amato TA, Landreneau RJ, McKenna RJ, Santos RS, Parker RJ. Prevalence of In Vitro Extreme Chemotherapy Resistance in Resected Nonsmall-Cell Lung Cancer. Ann Thorac Surg. 2006; 81(2): 440-7.

19. Eckardt JR, Bentsion DL, Lipatov ON, Polyakov IS, Mackintosh FR, Karlin DA, et al. Phase II Study of Picoplatin as Second-line Therapy for Patients with Small Cell Lung Cancer. J Clin Oncol. 2009; 27(12): 2046-51.

20. Mauer AM, Cohen EE, Ma PC, Kozloff MF, Schwartzberg L, Coates AI, et al. A Phase II Study of ABT-751 in Patients with Advanced Non-Small Cell Lung Cancer. J Thorac Oncol. 2008; 3(6): 631-6.

21. Verrils NM, Flemming CL, Liu M, Ivery MT, Cobon GS, Norris $\mathrm{MD}$, et al. Microtubule Alterations and Mutations Induced by Desoxyepothilone B: Implications for Drug-Target Interaction. Chem Biol. 2003; 10(7): 597-607.

22. Magnani N, Ortuso F, Soro S, Alcaro S, Tramontano A, Botta M. The $\beta I / \beta I I I-t u b u l i n$ Isoforms and Their Complexes with Antimitotic Agents. FEBS J. 2006; 273(14): 3301-10.

23. Jordan MA, Miller H, Ray A, Banerjee A, Manna T, Ni L, et al. The Pat-21 breast cancer model derived from a patient with primary Taxol resistance recapitulates the phenotype of its origin has altered $\beta$-tubulin expression and is sensitive to ixabepilone. Presented at the 97th AACR Annual Meeting. Washington, District of Colombia, April 1-5; 2006 [abstract LB-280].

24. Mitsudomi T, Kosaka T, Endoh H, Horio Y, Hida T, Mori S, et al. Mutations of the Epidermal Growth Factor Receptor Gene Predict Prolonged Survival After Gefitinib Treatment in Patients With NonSmall-Cell Lung Cancer With Postoperative Recurrence. J Clin Oncol. 2005; 23(11): 2513-20. 
25. Cheng L, Zhang S, Alexander R, Yao Y, MacLennan GT, Pan CX, et al. The Landscape of EGFR Pathways and Personalized Management of Nonsmall-Cell Lung Cancer. Future Oncol. 2011; 7(4): 519-41.

26. Keedy VL, Temin S, Somerfield MR, Beasley MB, Johnson DH, McShane LM, et al. American Society of Clinical Oncology Provisional Clinical Opinion: Epidermal Growth Factor Receptor (EGFR) Mutation Testing for Patients With Advanced Non- SmallCell Lung Cancer Considering First-Line EGFR Tyrosine Kinase Inhibitor Therapy. J Clin Oncol. 2011; 29(15): 2121-7.

27. Cataldo VD, Gibbons DL, Pérez-Soler R, Quintás-Cardama A. Treatment of Non-Small-Cell Lung Cancer with Erlotinib or Gefitinib. N Engl J Med. 2011; 364(10): 947-55.

28. Yang XD, Jia XC, Corvalan JR, Wang P, Davis CG, Jakobovits A. Eradication of Established Tumors by a Fully Human Monoclonal Antibody to the Epidermal Growth Factor Receptor without Concomitant Chemotherapy. Cancer Res. 1999; 59(6): 1236-43.

29. Brahmer J, Karen LR, Baas P, Crino L, Eberhardt WEE, Poddubskaya $\mathrm{E}$, et al. Nivolumab versus Docetaxel in Advanced Squamous-Cell Non-Small-Cell Lung Cancer. N Engl J Med. 2015; 373(17): 12335 .

30. Ribas A, Puzanov I, Dummer R, Schadendorf D, Hamid O, Robert $\mathrm{C}$, et al. Pembrolizumab versus Investigator-Choice Chemotherapy for Ipilimumab-Refractory Melanoma (KEYNOTE-002): A Randomised, Controlled, Phase 2 Trial. Lancet Oncol. 2015; 16: 908-18.

31. Dela Cruz CS, Tanoue LT, Matthay RA. Lung Cancer: Epidemiology, Etiology, and Prevention. Clin Chest Med. 2011; 32(4): 605-44.

32. Rades D, Käsmann L, Schild SE, Janssen SA. Survival Score for Patients Receiving Palliative Irradiation for Locally Advanced Lung Cancer. Clin Lung Cancer. 2016; 17(6): 558-62.

33. Reck M, Rodríguez-Abreu D, Robinson AG, Hui R, Csőszi T, Fülöp A, et al. Pembrolizumab versus Chemotherapy for PD-L1-Positive Non-Small-Cell Lung Cancer. N Engl J Med. 2016; 375(19): $1823-$ 33.

34. Li X, Asmitananda T, Gao L, Gai D, Song Z, Zhang Y, et al. Biomarkers in the Lung Cancer Diagnosis: A Clinical Perspective. Neoplasma. 2012; 59(5): 500-7.

35. Meiliana A, Dewi NM, Wijaya A. Cancer Immunotherapy: A Review. Indones Biomed J. 2016; 8: 1-20.

36. Chae YK, Pan A, Davis AA, Raparia K, Mohindra NA, Matsangou $\mathrm{M}$, et al. Biomarkers For PD-1/PD-L1 Blockade Therapy in NonSmall-Cell Lung Cancer: Is PD-L1 Expression A Good Marker For Patient Selection?. Clin Lung Cancer. 2016; 17(5): 350-61.

37. Meiliana A, Dewi NM, Wijaya A. Personalized Medicine: The Future of Health Care. Indones Biomed J. 2016; 8: 127-46.

38. Mok TS, Wu YL, Thongprasert S, Yang CH, Chu DT, Saijo N, et al. Gefitinib or Carboplatin-Paclitaxel in Pulmonary Adenocarcinoma. N Engl J Med. 2009; 361: 947-57.

39. Jackman DM, Miller VA, Cioffredi LA, Yeap BY, Jänne PA, Riely GJ, et al. Impact of Epidermal Growth Factor Receptor and KRAS Mutations on Clinical Outcomes in Previously Untreated NonSmall Cell Lung Cancer Patients: Results of An Online Tumor Registry of Clinical Trials. Clin Cancer Res. 2009; 15(16): 5267-73.

40. Pao W, Wang TY, Riely GJ, Miller VA, Pan Q, Ladanyi M, et al. KRAS Mutations and Primary Resistance of Lung Adenocarcinomas to Gefitinib or Erlotinib. PLoS Med. 2005; 2(1): e17. doi: 10.1371/ journal.pmed.0020017

41. Thomas RK, Baker AC, Debiasi RM, Winckler W, Laframboise T,
Lin WM, et al. High-throughput Oncogene Mutation Profiling in Human Cancer. Nat Genet. 2007; 39(3): 347-51.

42. Linardou H, Dahabreh IJ, Kanaloupiti D, Siannis F, Bafaloukos D, Kosmidis P, et al. Assessment of Somatic k-RAS Mutations as A Mechanism Associated with Resistance to EGFR-Targeted Agents: A Systematic Review and Meta-Analysis of Studies in Advanced Non-Small-Cell Lung Cancer and Metastatic Colorectal Cancer. Lancet Oncol. 2008; 9(10): 962-72.

43. Mao C, Qiu LX, Liao RY, Du FB, Ding H, Yang WC, et al. KRAS Mutations and Resistance to EGFR-TKIs Treatment in Patients with Non-Small Cell Lung Cancer: A Meta-Analysis of 22 Studies. Lung Cancer. 2011; 69(3): 272-8.

44. Eberhard DA, Johnson BE, Amler LC, Goddard AD, Heldens SL, Herbst RS, et al. Mutations in the Epidermal Growth Factor Receptor and in KRAS Are Predictive and Prognostic Indicators in Patients With Non-Small-Cell Lung Cancer Treated With Chemotherapy Alone and in Combination With Erlotinib. J Clin Oncol. 2005; 23(25): 5900-9.

45. De Roock W, Claes B, Bernasconi D, De Schutter J, Biesmans B, Fountzilas G, et al. Effects of KRAS, BRAF, NRAS, and PIK3CA Mutations on The Efficacy of Cetuximab Plus Chemotherapy in Chemotherapy-Refractory Metastatic Colorectal Cancer: A Retrospective Consortium Analysis. Lancet Oncol. 2010; 11(8): 753-62.

46. Vakiani E, Solit DB. KRAS and BRAF: Drug Targets and Predictive Biomarkers. J Pathol. 2011; 223(2): 219-29.

47. Davies H, Bignell GR, Cox C, Stephens P, Edkins S, Clegg S, et al. Mutations of The BRAF Gene in Human Cancer. Nature. 2002; 417: 949-54.

48. Schmid K, Oehl N, Wrba F, Pirker R, Pirker C, Filipits M. EGFR/ KRAS/BRAF Mutations in Primary Lung Adenocarcinomas and Corresponding Locoregional Lymph Node Metastases. Clin Cancer Res. 2009; 15(14): 4554-60.

49. Ladanyi M, Pao W. Lung Adenocarcinoma: Guiding EGFR-Targeted Therapy and Beyond. Mod Pathol. 2008; 21(Suppl 2): S16-S22. doi: 10.1038/modpathol.3801018.

50. Seki T, Hagiya M, Shimonishi M, Nakamura T, Shimizu S. Organization of the Human Hepatocyte Growth Factor-Encoding Gene. Gene 1991; 102(2): 213-9.

51. Nguyen KS, Kobayashi S, Costa DB. Acquired Resistance to Epidermal Growth Factor Receptor Tyrosine Kinase Inhibitors in Non-Small-Cell Lung Cancers Dependent on the Epidermal Growth Factor Receptor Pathway. Clin Lung Cancer. 2009; 10(4): 281-9.

52. Beau-Faller M, Ruppert AM, Voegeli AC, Neuville A, Meyer N, Guerin E, et al. MET Gene Copy Number in Non-small Cell Lung Cancer: Molecular Analysis in a Targeted Tyrosine Kinase Inhibitor Naïve Cohort. J Thorac Oncol. 2008; 3(4): 331-9.

53. Engelman JA, Zejnullahu K, Mitsudomi T, Song Y, Hyland C, Park $\mathrm{JO}$, et al. MET Amplification Leads to Gefitinib Resistance in Lung Cancer by Activating ERBB3 Signaling. Science. 2007; 316(5827): 1039-43.

54. Eder JP, Vande Woude GF, Boerner SA, LoRusso PM. Novel Therapeutic Inhibitors of the c-Met Signaling Pathway in Cancer. Clin Cancer Res. 2009; 15(7): 2207-14.

55. Gandara DR, Grimminger P, Mack PC, Lara PN Jr, Li T, Danenberg $\mathrm{PV}$, et al. Association of Epidermal Growth Factor Receptor Activating Mutations with Low ERCC1 Gene Expression in Non- 
small Cell Lung Cancer. J Thorac Oncol. 2010; 5(12): 1933-8.

56. Gomez-Roca C, Raynaud CM, Penault-Llorca F, Mercier O, Commo F, Morat L, et al. Differential Expression of Biomarkers in Primary Non-small Cell Lung Cancer and Metastatic Sites. J Thorac Oncol.
2009; 4(10): 1212-20.

57. Cheng L, Alexander RE, Maclennan GT, Cummings OW, Montironi R, Lopez-Beltran A, et al. Molecular Pathology of Lung Cancer: Key to Personalized Medicine. Mod Pathol. 2012; 25(3): 347-69. 